\title{
Are the Cells Stronger than we Think?
}

\author{
Silvia Hervas-Raluy ${ }^{1}, \mathrm{M}^{\mathrm{a}}{ }{ }$ osé Gomez-Benito ${ }^{1}$, Carlos Borau-Zamora ${ }^{1}$, José Manuel \\ Garcia-Aznar $^{1}$ \\ ${ }^{1}$ Multiscale in Mechanical and Biomedical Engineering (M2BE) \\ Instituto de Investigación en Ingeniería de Aragón (I3A) \\ Universidad de Zaragoza, Mariano Esquillor s/n, 50018, Zaragoza, Spain. \\ Tel. +34-976762707, e-mail: hervas@unizar.es
}

\begin{abstract}
This work presents a novel methodology to calculate the traction forces exerted by the cell in a threedimensional (3D) Traction Force Microscopy (TFM) set-up. This methodology starts from the images taken in the TFM essay. In addition, the finite strains hypothesis is assumed in order to capture the cell behaviour.
\end{abstract}

\section{Methods}

Physical forces exerted by cells regulate many cellular processes such as angiogenesis, wound healing and cancer metastasis. Due to the key role of such forces, it is essential to achieve a more complete knowledge of cell traction force regulation as well as to be able to measure these forces.

The aim of the TFM experiments is to recover the force exerted by the cell in its movements. In this problem the known configuration is the deformed one (the final position and shape of the cell) and the displacements of the cell. Thus, it becomes necessary to solve an inverse problem. Unlike traditional problems (direct ones), in which the known configuration is the undeformed one and the force field map is also known, inverse problems consider these as the unknowns. Therefore, the main challenge of this work is to recover the undeformed configuration and also the cell traction force maps. To this end, images taken in the 3D-TFM experiment are processed to obtain both the cell voxelised geometry and the displacements field. These data are the input of the inverse problem, which is solved via Finite Element Method (FEM). The analysis is iterative, in which each iteration is composed of three analyses. Both extra-cellular matrix (ECM) and cell are discretised in the finite element mesh. The proposed methodology allows defining any type of material for any domain. In the present work, it is assumed that both cell and ECM behave as an hyperelastic Neohookean materials.

\section{Results and discussion}

To validate the presented methodology, a direct problem has been created using FEM. Thus, a set of known forces have been applied on the tested geometry, a sphere. The deformation obtained in the direct problem, and thus the one to recover, is above $50 \%$. Total cell displacements recovered are depicted in Figure 1. In order to be able to recover such dramatic changes in shape, the methodology adopts an updated Lagrangian approximation. Therefore, the geometry is updated as the analysis converges to the solution, and so does the FE mesh. Towards to obtain the surface traction vectors field in the final configuration (Figure 2), these traction vectors have to be calculated in every intermediate configuration. This constitutes an upgrade of the methodology proposed by Peñas et. al. [1] which did not recover the cell traction field.

The computation of 3D traction forces presents a far more complicated problem than the two-dimensional ones. However, this becomes absolutely necessary since cell behaviour greatly depends on dimensionality [2]. Legant et al. [3] opens the way to recover traction forces in $3 \mathrm{D}$ analysis, even though they use a linear elastic material approximation. The methodology proposed in the present work underlies on a nonlinear material behaviour, using the Neohookean material formulation.

In TFM experiments, cell undergoes significant displacements, therefore, the use of finite strains formulation is really important. However, the implementation of this hypothesis is not trivial, and therefore other methodologies are limited to linear strains $[3,4]$.

\section{Conclusions}

The proposed methodology allows quantifying traction forces exerted by the cell. In contrast to other methodologies present in the literature, the one proposed here is fully automatic. The input are the images taken during the TFM experiment, obtaining

Revista “Jornada de Jóvenes Investigadores del I3A”, vol. 8 (Actas de la IX Jornada de Jóvenes Investigadores del I3A -11 de diciembre de 2020). ISSN 2341-4790. 
directly the traction forces at the cell surface. Therefore, this methodology is conceived as a useful tool to complement the laboratory experiments and extract results in an automatized way. In order to facilitate this, the methodology is applicable to any cellular type, since it is independent on the cell geometry. Furthermore, materials with hyperelastic behaviour have been modelled, although it is clearly also applicable to models that can be simplified with other constitutive model assumptions.

\section{Acknowledgements}

This research was supported by the Spanish Ministry of Economy and Competitiveness (Grant RTI 2018094494-B-C21) and by the Regional Government of Aragon (Grant 2019/23). The financial support of the European Union through the European Research Council (project ERC-2016-PoC 737543) is gratefully acknowledged.

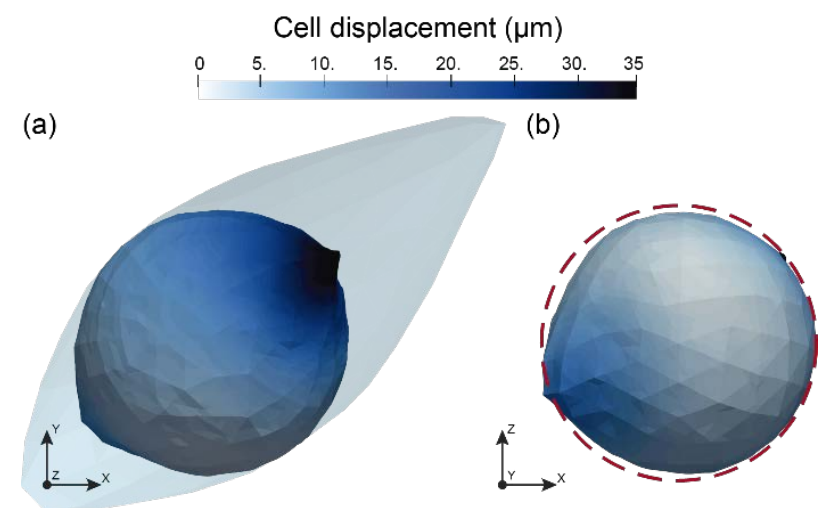

Figure 1: Cell displacement $(\mu \mathrm{m})$. (a) XY view. The undeformed configuration is shown backwards in light blue, which is considerably bigger than the final configuration. The greatest displacement is located in the upper right area. (b) XZ view. Red dashed line indicates the target configuration, that is, the initial (undeformed) configuration of the direct problem.

\section{REFERENCIAS}

[1]. JORGE-PEÑAS, Alvaro, et al. 3D full-field quantification of cell-induced large deformations in fibrillar biomaterials by combining non-rigid image registration with label-free second harmonic generation. Biomaterials, 2017, 136, 86-97. Available from: doi: /10.1016/j.biomaterials.2017.05.015

[2]. PAMPALONI, Francesco; REYNAUD, Emmanuel G.; STELZER, Ernst HK. The third dimension bridges the gap between cell culture and live tissue. Nature reviews Molecular cell biology, 2007, 8(10), 839-845. Available from: doi: 10.1038/nrm2236

[3]. LEGANT, Wesley R., et al. Measurement of mechanical tractions exerted by cells in threedimensional matrices. Nature methods, 2010, 7(12), 969-971. Available from: doi: 10.1038/nmeth.1531.

[4]. PALACIO, Javier, et al. Numerical estimation of 3D mechanical forces exerted by cells on non-linear materials. Journal of biomechanics, 2013, 46(1), 5055. Available from: doi: 10.1016/j.jbiomech.2012.10.009

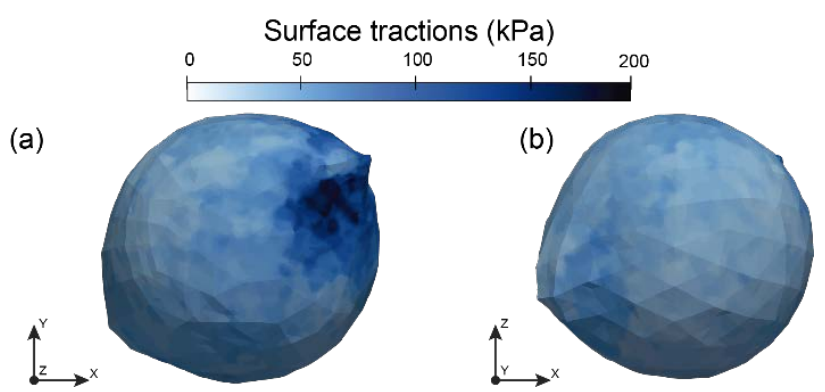

Figure 2: Recovered surface tractions (kPa). (a) XY view. The maximum surface tractions are located in the upper right area, where the cell is suffering the greatest displacements. (b) XZ view. Tractions are lower and more uniform in the rest of the cell, although a slight increase can be appreciated in the lower left area. 